\title{
Primary care genomic research: One eye on the future
}

Professor Philip H Evans GP and Associate Professor of General Practice and Primary Care, University of Exeter Medical School and National Specialty Lead for Primary Care, NIHR CRN Email: p.h.evans@exeter.ac.uk

Dr Imran Rafi GP and Senior Lecturer, St George's University of London, London

Dr Judith Hayward GP, Affinity Care and GPwSI in Genetics, Yorkshire Regional Genetics Service

Dr Molly Dineen Interim Foundation Doctor, Royal Cornwall Hospitals NHS Trust

Dr Fiona M. Walter GP and Reader in Primary Care Cancer Research, University of Cambridge

\section{Primary care genomic research: one eye on the future}

The exceptional advances in genomic medicine in the last two decades, since the sequencing of the human genome, have already had a major impact on healthcare in the UK. Until recently this was predominantly in secondary and tertiary care but we must now integrate "mainstream" genomics in primary care. So far integration has of necessity been empirical but must progress towards implementation research. We describe recent work undertaken by the RCGP and the NIHR CRN outlining areas where research is needed to fully integrate genomics into routine primary care and secure the future of these exciting developments.

\section{Clinical case scenario}

A 42-year-old man attends an NHS health check with a healthcare assistant (HCA) which includes a blood test for $\mathrm{HbA1c}$, lipid profile and genomic profiling for cardiovascular disease (CVD) risk. During the consultation the HCA asks about his family history of CVD, including first- and second-degree relatives, and records this in the electronic health record (EHR). The patient then has a follow-up phone consultation with his GP who uses a current version of QRISK to generate a Polygenic Risk Score (PRS) for CVD over the next 10 years. Data regarding genomic variants are extracted and incorporated with other data including smoking and family history. The patient's PRS is above the threshold for offering primary prevention with a statin, and he asks if the genomic test is 'the one to see if statins won't agree with me'. The GP explains that this genomic test only identifies variants which influence his CVD risk; however, he knows that the practice is recruiting patients for a research study aiming to evaluate pharmacogenomic testing delivered by the practice pharmacist and asks if the patient would be interested in receiving further information.

\section{Background}

Since the publication of the 'Generation Genome' 2016 Annual Report by the Chief Medical Officer Dame Sally Davies in 2017, there has been a burgeoning increase in the integration of genomic medicine into UK clinical care (Davies, 2017). Large-scale healthcare 
reorganisation has already commenced, with the creation of a new nationally commissioned Genomic Medicine Service (GMS) supported by seven Genomic Laboratory Hubs, soon to be overtaken by the new GMS Alliances. These initiatives have all arisen from the internationally renowned 100,000 Genomes project, focusing on genomic testing for patients with cancer, infectious diseases and as yet undiagnosed rare disorders including developmental disorders (Turnbull et al., 2018). The 100,000 Genomes Project has also supported a large number of empirical research studies further developing our understanding of pathological variants in the context of these disorders. This whole initiative has been led by NHS England (NHS England, 2016a).

Clinicians in secondary and tertiary care, particularly in oncology, are now experienced at using genomic variation as the basis for a personalised approach to assessing risk for individual cancers and cancer subtypes. This fits with the desire to promote personalised

medicine- "the right drug, at the right dose, to the right patient, at the right time" - and with NHS England's "4Ps" programme of promoting individualised or personalised medicine in all aspects of healthcare (NHS England, 2016b). It is likely, as some commentators suggest, that the whole taxonomy of medicine will change as the genomic basis of disease becomes clearer. We are already seeing examples of this in diabetes, for example, where genomic subtypes of diabetes have been suggested, with different phenotypes, clear genotypic differences and very different disease trajectories (Ahlqvist et al., 2018). However, in other subtypes of diabetes such as maturity onset diabetes of the young (MODY) the genomic basis is much clearer with genomic mutations affecting the potassium channel now prompting urgent changes of treatment for diabetes ( (Delvecchio et al., 2020).

The patient perspective on genomic testing has become extremely important of late, particularly when direct-to-consumer (DTC) testing has flourished (Horton et al., 2019). GPs can expect to see increasing requests from patients to advise on their DTC genomic results, which may prove a challenge to most primary care professionals. The RCGP and British Society of Genetic Medicine (BSGM) have recently produced helpful advice on DTC testing (RCGP, 2019). As the genomic revolution in healthcare moves away from patients referred into secondary and tertiary care towards primary care, it is clear that there are a large number of unanswered questions. A recent Lancet commentary (Manolio et al., 2019), identified three main challenges for genomic medicine implementation which will need to be addressed (Box 1).

\section{BOX 1 HERE}

As the need for implementation of genomic medicine in primary care has become more apparent, a number of key educational and implementation initiatives have been led by the RCGP and Health Education England. It is against this background that the RCGP and the Clinical Research Network (CRN) of the National Institute of Health Research (NIHR) came together. The aim was to identify and define the key building blocks that were needed in primary care to establish a research evidence-base in primary care to facilitate the delivery of genomic medicine. 
In 2018 the RCGP and the CRN engaged in a collaborative process led through Cluster C of the CRN, based in King's College, London. This process was supported by key stakeholders and national leaders in genomics as well as front-line clinicians in primary care and representatives from a number of the 15 local Clinical Research Networks (CRNs) across England.

Following initial meetings, and on the advice of the Steering Group, it became clear that there were three main areas or workstreams that needed further exploration, namely, informatics and family history taking, education of GPs and primary care staff, and operationalising genomic research. For each of these key workstreams a meeting was held with stakeholders and individuals with an interest in that area. A number of recommendations were identified to further facilitate effective primary care research in genomics (RCGP and NIHR CRN, 2018).

\section{Challenges}

\section{Informatics and family history recording}

During the consultation process the stakeholders identified one of the biggest barriers to effective primary care genomics research in the NHS to be the lack of standardised family history recording in the primary care electronic health records (EHRs). Furthermore, there were major difficulties with recording of specific genomic variants in the EHR.

For many decades now, GPs have known the importance of family history in the health of the patient (Qureshi et al., 2012) and acknowledged its relevance to family practice across the world (Vorderstrasse et al., 2013) (Walter and Emery, 2012). However, the level and quality of family history recording in GP records, both in the UK and abroad, is poor (Dhiman et al., 2014) (Wilson et al., 2009) and is inadequate to provide reliable disease risk assessments. This is despite various approaches and attempts to develop short family history tools (Walter et al., 2013) (Emery et al., 2014) and evidence of their successful implementation in routine clinical practice (Qureshi et al., 2012).

In the recent Lancet series on genomic medicine Ginsburg and colleagues (Ginsburg et al., 2019) confirmed that family history "is (the) most useful means of assessing risk for common chronic diseases" and was often underused for actionable risk assessment. In their opinion, family history remains the gold standard for risk assessment and advances in personalised medicine must align with improvements in family history recording. Importantly, they recognised that family history reporting, particularly in a primary care context, identifies multiple aspects of disease risk, including environmental, molecular and social. The 2018 RCGP/CRN report proposes a practical approach to clinical genetics and genomics for primary care, acknowledging the importance of systematic family history recording for disease risk assessment to underpin the recruitment and stratification of patients into research studies.

Many studies have shown that there is not yet an optimal tool for family history recording in primary care with issues around digital data collection and storage, updating and 
confidentiality (De Hoog et al., 2014). However, there have been a number of recent disease-specific UK research innovations in primary care risk assessment tools. The FAMCAT clinical case-finding algorithm has been developed and evaluated to identify patients with familial hypercholesterolaemia in primary care (Weng et al., 2018). The CanRisk Tool (Archer et al., 2020) incorporates the new version of BOADICEA, the Breast and Ovarian Analysis of Disease Incidence and Carrier Estimation Algorithm, into a tool for use in primary care and in genetics clinics (Lee et al., 2019).

To optimise use, these and other disease risk assessment tools ideally need to be integrated into the clinical system operated by the GP software suppliers with an associated Clinical Decision Support System (Chase et al., 2017). The tool itself would need to be associated with new educational resources for primary care staff to enable optimal recording of family history, risk assessment and tailored management including evidence-based communication.

Other information technology issues raised by the group included the need to facilitate better recording of detailed information, for example single gene variants, which would assist in the diagnosis of disorders with a genomic basis. This may be facilitated by the imminent move from Read codes to SNOMED codes in GP systems. Additionally, there will be a need to record pharmacogenomic variants (see below) as the impact of genomics on prescribing in a primary care setting could increase exponentially in the near future.

Another issue that affects both primary and secondary care genomics is the recording of family members' diseases in the medical record of the patient who is the case. Ironically, although GPs are family doctors and used to dealing with families, there may be uncertainty about recording family history in the family members' records, for reasons of medical confidentiality coupled with the fact that patients can now access their own medical records and could inadvertently see the FH of family members.

One solution to this is to use the household function which is available in some GP clinical systems and is designed for administrative purposes. However, patients living at the same address may not be in the same family. In the 100,000 Genomes project some hospitals have instituted a family number to assist with this. Clear guidance does exist (Royal College of Physicians, Royal College of Pathologists and British Society for Genetic Medicine, 2019) as does the concept of the "joint account" which some GPs may find helpful (Parker and Lucassen, 2004).

\section{Educational needs}

In order to facilitate effective primary care genomic research, the stakeholders recognised the need for genomics education crosscutting both of the other workstreams. Appropriate education of healthcare professionals is generic to all initiatives in primary care and genomics research is no different. The HEE's Genomics Education Programme (GEP) has already started to identify the key knowledge (in particular knowledge updates) and skills required by GPs in this area. This will apply to both GPs with and without an extended role 
in primary care genomics. This work has been conducted in the context of a wider educational needs assessment for genomics in primary care.

This has included a multidisciplinary Delphi-style survey carried out by HEE GEP in 2017 aimed to identify knowledge, skills and attitudes required by GPs in the genomics era. The themes identified broadly fell into 3 categories:

- Knowledge, Skill and Attitudes (KSA) which were core to General Practice. These include communication skills and risk communication and are captured in the RCGP core curriculum document

- Pre-existing KSA in genetics which are generalisable, e.g. family history assessment and reproductive health options

- Novel KSA to the genomics era, e.g. understanding of NHS GMS, eligibility for genomic tests, and ability to communicate genomic information, as well as some novel ethical issues in and around data

The RCGP (2019) have produced a genomics toolkit with a section that includes helpful resources to help educate primary care staff engaged in genomics research. A grounding in genomic education is particularly important in the research context where it is likely that GPs and their teams may be consenting patients to participate in genomic studies and likewise may be feeding back the results of genomic tests. Both aspects of this research activity need an appropriate level of genomic understanding and of the implications of positive/negative genomic testing, in whatever context the study is placed.

The report recommended that the NIHR CRN develop specific training addressing these issues around genomic research and that study-specific educational material would help deliver genomic studies moving forward. This would build on the excellent work already undertaken by the NIHR CRN in the wider context of stratified medicine studies and resources developed to underpin that initiative (Attar et al., 2019).

\section{Operationalising NIHR genomic research in primary care}

The stakeholder group agreed that genomic studies are not completely similar to studies of therapeutic interventions or drug treatment. The complexity of genomic testing, coupled with the implications for the patient and their family, adds another dimension to a primary care research study recruiting through general practices or pharmacies. An example of a study that recruited from General Practice and the NIHR-CRN was the BARCODE study, looking at targeted genetic profiling for prostate cancer (Case Medical Research, 2019)

This workstream considered a number of similar case studies from primary care genomic research already underway and identified key issues around the taking and storage of genomic specimens, the cost of genomic testing and recording results from research studies in the EHR. The group also noted that study teams would need to provide more education to practices undertaking genomic research. This could be in the form of 'academic detailing' visits by the study team to promote the study to practices and answer questions in a faceto-face setting. 
The Report recommended that the CRN costing processes are refined; case studies of successful CRN genomic research studies are developed to both promote research and identify key learning from other studies; CRN staff facilitating genomic studies should have a good understanding of genomics, and finally that the associated paperwork should be minimal.

\section{Current areas of activity in primary care genomic research}

The NIHR CRN recently (November 2019) invited the leading UK academics in primary care genomic research to a Clinical Studies Group to identify their current areas of interest and research activity. The following areas were identified as the current strengths:

\section{Pharmacogenomics}

The role of genomic variants in determining the best way to individualise drug therapy is increasingly recognised, although the evidence-base for clinical utility in a primary care setting has not yet been demonstrated, nor has the cost-effectiveness. A recent Lancet paper (Roden et al., 2019) is a helpful summary of the current state of play but also identifies a number of potential barriers to implementation, some of which have been mentioned previously, such as the current EHR systems and the challenges of costeffectiveness. Another recent qualitative paper also explores these issues (Rafi et al, 2020).

\section{Rare diseases}

Work in the 100,000 Genomes project showed the clinical utility of genomic testing in rare diseases, including confirming a diagnosis, establishing prognosis, potentially changing treatment and enabling accurate genetic counselling for the family. In the Deciphering Developmental Disorders (DDD) study an extra $10 \%$ of patients received a definitive diagnosis through a genomic-driven approach (Fitzgerald et al, 2015). It is often not recognised that patients with rare disease constitute one in 17 of the GP population (Department of Health, 2013) and GPs have a positive role to play in the diagnosis, management and referral of these patients, including the use of appropriate genomic testing.

Building on the excellent work undertaken by the 100,000 Genomes project, there is now a move to undertake further research in primary care using large GP databases to determine phenotypic information that could potentially identify those patients in practices with rare diseases. This could build on tools developed through artificial intelligence, machine learning and predictive modelling. Although this is in its infancy, the potential in this area is exceptional, building on the renowned strengths of the primary care GP clinical database in the UK (Saunders et al., 2020). There are also linked initiatives to identify phenotypic and genotypic information that could be used to identify high-risk individuals in whom adverse reactions to medication could be predicted with greater certainty. 


\section{Polygenic risk scores}

There has been a rapid proliferation of polygenic risk score reports, particularly those used in the context of common chronic diseases in primary care such as diabetes, CVD, depression and psychiatric disorders. GPs are used to working with predictive risk tools and communicating risk to patients, but the additional benefit of these tools above and beyond existing risk calculators will need to be clarified in further research. The PHG foundation published an excellent review of polygenic risk scores in 2019 (PHG, 2019).

\section{Family history and risk prediction tools}

A number of UK primary care academic centres are engaged in developing, testing and researching the implementation of integrated disease risk assessment tools, for example to facilitate the early diagnosis of Familial Hyperlipidaemia in Nottingham (Weng et al., 2015) and inherited cancers in Cambridge (Lee et al., 2019).

\section{Risk stratification in cancer}

The BARCODE study (Case Medical Research, 2019), led by the Institute for Cancer Research in London, has recruited from primary care and is investigating using genetic and genomic profiling to stratify men for targeted population screening for prostate cancer. Following a pilot study, a larger study is recruiting 5000 men aged between 55 and 69. The NIHR CRN has successfully facilitated recruitment.

\section{The future}

It is clear that there are a large number of research studies to be undertaken and the NIHR CRN is adapting to facilitate these studies as they come onto the CRN portfolio. As new genetic mutations and genomic variants are identified, it is vital that they are incorporated into approaches that provide personalised screening, early detection, diagnosis, management and prescribing and that validation is undertaken in a primary care setting. Perhaps the greatest research needed moving forward is for implementation research. Possible research questions include: how best should genomic medicine be embedded within primary care, what is the optimum model for referral and cost-effectiveness, and how best to engage primary care teams with new knowledge needed to deliver personalised medicine?

The NHS Long Term Plan (NHS Improvement, 2019) is explicit in its support for genomic implementation and genomic research in the NHS. The 2018 RCGP/CRN Report recommended that funding bodies such as UKRI and the NIHR consider the need for funding of high-quality implementation studies around genomic initiatives in primary care as a matter of priority in the next few years.

As well as the need for better genomic education medical school and postgraduate training in general practice to empower the future workforce, there is also a need for GP registrars and early career GPs to be provided with opportunities to pursue an academic career in 
primary care genomic research. This could be part of the extensive career development and support funding that is provided by the NIHR Academy Fellowship Programme for all stages of academic career development (NIHR Academy, 2020). 


\section{Summary}

Recent developments in genetics and genomics are certain to impact on primary care as the pace of change continues to accelerate. There is therefore an urgent need for multi-faceted research studies to determine the evidence-base that is needed to ensure successful implementation of genomic medicine in a primary care setting.

\section{Key Points:}

- The integration of genomics into routine primary care needs an understanding of how to use genomics in a personalised approach to management of patients, including new treatments and a new infrastructure now led by the nationally commissioned Genomic Medicine Service

- Integration is being helped, informed and promoted by the Royal College of General Practitioners (RCGP), the Genomics Education Programme and Health Education England's online genomics resources

- Integration is being informed and helped by research into informatics, education and operationalising genomic research in primary care as identified by the RCGP and the National Institute of Health Research Clinical Research Network

- Integration will involve recognition that a genomic result may have implications for all family members, whether or not they need screening; if possible the GP should work with the whole family as well as the individual

- Primary care-based genomic research is needed to provide the evidence base, including clinical utility and cost-effectiveness which will underpin the use of genomics in primary care 


\section{References and further information}

Ahlqvist E, Storm P, Käräjämäki A, et al. (2018) Novel subgroups of adult-onset diabetes and their association with outcomes: a data-driven cluster analysis of six variables. The Lancet Diabetes and Endocrinology, 6(5):361-369 DOI: 10.1016/S2213-8587(18)30051-2.

Archer S, De Villiers CB, Scheibl F, et al. (2020) Evaluating clinician acceptability of the prototype CanRisk tool for predicting risk of breast and ovarian cancer: A multimethods study. PLOS ONE, 15(3):e0229999 DOI: 10.1371/journal.pone.0229999.

Attar SG, Poustie VJ, Smye SW, et al. (2019) Working together to deliver stratified medicine research effectively. In: British Medical Bulletin, 129(1):107-116 DOI: 10.1093/bmb/ldz003.

Case Medical Research (2019) The BARCODE 1 Study (Full Study): The Use of Genetic Profiling to Guide Prostate Cancer Targeted Screening. DOI: 10.31525/ct1nct03857477.

Chase DA, Baron S and Ash JS (2017) Clinical decision support and primary care acceptance of genomic medicine. In: Studies in Health Technology and Informatics, pp700-703 DOI: 10.3233/978-1-61499-830-3-700.

Davies SC (2017) Annual Report of the Chief Medical Officer 2016. Available at: www.gov.uk/government/publications/chief-medical-officer-annual-report-2016generation-genome (accessed 19 October 2020).

De Hoog CLMM, Portegijs PJM and Stoffers HEJH (2014) Family history tools for primary care are not ready yet to be implemented. A systematic review. European Journal of General Practice, 20(2):125-33 DOI: 10.3109/13814788.2013.840825.

Delvecchio M, Pastore C and Giordano P (2020) Treatment Options for MODY Patients: A Systematic Review of Literature. Diabetes Therapy. DOI: 10.1007/s13300-020-00864-4.

Department of Health (2013) The UK Strategy for Rare Diseases. Department of Health and Social Care Publication.

Dhiman P, Kai J, Horsfall L, et al. (2014) Availability and quality of coronary heart disease family history in primary care medical records: Implications for cardiovascular risk assessment. PLoS ONE, 9(1):e81998 DOI: 10.1371/journal.pone.0081998.

Emery JD, Reid G, Toby Prevost A, et al. (2014) Development and validation of a family history screening questionnaire in Australian primary care. Annals of Family Medic ine, 12(3):241-249DOI: 10.1370/afm.1617.

Ginsburg GS, Wu RR and Orlando LA (2019) Family health history: underused for actionable risk assessment. The Lancet, 394(10198):596-603 DOI: 10.1016/S0140-6736(19)312759.

Horton R, Crawford G, Freeman L, et al. (2019) Direct-to-consumer genetic testing. The BMJ 367:I5688 DOI: 10.1136/bmj.I5688.

Lee A, Mavaddat N, Wilcox AN, et al. (2019) BOADICEA: a comprehensive breast cancer risk 
prediction model incorporating genetic and nongenetic risk factors. Genetics in Medicine,21(8):1708-1718 DOI: 10.1038/s41436-018-0406-9.

Lesko $\amalg$, Zineh I and Huang SM (2010) Editorial: What is clinical utility and why should we care? Clinical Pharmacology and Therapeutics, 88(6):729=733 DOI: 10.1038/clpt.2010.229.

Manolio TA, Rowley R, Williams MS, et al. (2019) Opportunities, resources, and techniques for implementing genomics in clinical care. The Lancet, 394(10197):511-520 DOI: 10.1016/S0140-6736(19)31140-7.

NHS England (2016a) 100,000 Genomes Project: Paving the way to Personalised Medicine. Available at: www.england.nhs.uk/publication/100000-genomes-project-paving-theway-to-personalised-medicine (accessed 19 October 2020).

NHS England (2016b) Improving Outcomes Through Personalised Medicine. Available at: www.england.nhs.uk/wp-content/uploads/2016/09/improving-outcomespersonalised-medicine.pdf (accessed 19 October 2020)

NHS Improvement (2019) NHS Long Term Plan Implementation Framework. Available at: www.longtermplan.nhs.uk/implementation-framework/ (accessed 19 October 2020)

NIHR Academy (2020). Integrated Academic Training. Available at: www.nihr.ac.uk/explorenihr/academy-programmes/integrated-academic-training.htm (accessed 23 May 2020)

Parker M and Lucassen AM (2004) Genetic information: A joint account? BMJ. DOI: 10.1136/bmj.329.7458.165.

PHG Foundation (2019) Polygenic scores, risk and cardiovascular disease. Cambridge: PHG Foundation. Available at: www.phgfoundation.org/report/polygenic-scorescardiovascular (accessed 19 Oct 2020).

Qureshi N, Armstrong S, Dhiman P, et al. (2012) Effect of adding systematic family history enquiry to cardiovascular disease risk assessment in primary care: A matched-pair, cluster randomized trial. Annals of Internal Medicine, 156(4):253-262 DOI: 10.7326/0003-4819-156-4-201202210-00002.

Rafi I, Crinson I, Dawes M, et al. (2020) The implementation of pharmacogenomics into UK General Practice: a qualitative study exploring barriers, challenges and opportunities, Journal of Community Genetics. In press. DOI: 10.1007/s12687-020-00468-2.

Roden DM, McLeod HL, Relling MV, et al. (2019) Pharmacogenomics. The Lancet, 394(10197):521-532 DOI: 10.1016/S0140-6736(19)31276-0.

RCGP, NIHR Clinical Research Network (2018) Preparing Primary and Community Care in the NHS for Genomic Research. Available at: www.nihr.ac.uk/documents/showcase/primary-care/rcgp-crn-genomics-researchreport.pdf. Accessed 23 May 2020.

RCGP (2019). Available at: www.rcgp.org.uk/clinical-andresearch/resources/toolkits/genomics-toolkit.aspx Accessed 23 May 2020 
RCGP, British Society for Genetic Medicine (2019). Position statement on direct to consumer testing. Available at: www.rcgp.org.uk/-/media/Files/CIRC/Clinical-Policy/Positionstatements/RCGP-position-statement-on-direct-to-consumer-genomic-testing-oct2019.ashx?la=en. Accessed 23 May 2020.

\section{RCGP. Clinical topic guide: Genomic medicine. Available at: www.rcgp.org.uk/training-} exams/training/gp-curriculum-overview.aspx (accessed 21 June 2020)

Royal College of Physicians, Royal College of Pathologists and British Society for Genetic Medicine (2019). Consent and confidentiality in genomic medicine: Guidance on the use of genetic and genomic information in the clinic. 3rd edition. Report of the Joint Committee on Genomics in Medicine. London. Available at: www.rcplondon.ac.uk/projects/outputs/consent-and-confidentiality-genomic-medicine (accessed 24 September 2020).

Saunders CL, Kilian B, Thompson DJ, et al. (2020) External validation of risk prediction models incorporating common genetic variants for incident colorectal cancer using UK Biobank. Cancer Prevention Research, DOI: 10.1158/1940-6207.capr-19-0521.

Fitzgerald TW, Gerety SS, Hurles ME (2015) Large-scale discovery of novel genetic causes of developmental disorders. Nature. DOI:10.1038/nature14135

Turnbull C, Scott RH, Thomas E, et al. (2018) The 100000 Genomes Project: Bringing whole genome sequencing to the NHS. BMJ (Online). 361:k1687DOI: 10.1136/bmj.k1687.

Vorderstrasse AA, Cho A, Voils $\mathrm{Cl}$, et al. (2013) Clinical utility of genetic risk testing in primary care: The example of Type 2 diabetes. Personalized Medicine, 10(6):549-563 DOI: $10.2217 /$ pme.13.47.

Walter FM and Emery JD (2012) Genetic advances in medicine: Has the promise been fulfilled in general practice? British Journal of General Practice, 62(596):120-121 DOI: 10.3399/bjgp12X629955.

Walter FM, Prevost AT, Birt L, et al. (2013) Development and evaluation of a brief selfcompleted family history screening tool for common chronic disease prevention in primary care. British Journal of General Practice 63(611):e393-400 DOI: 10.3399/bjgp13X668186.

Weng S, Kai J, Tranter J, et al. (2018) Improving identification and management of familial hypercholesterolaemia in primary care: Pre- and post-intervention study. Atherosclerosis, 274:54-60 DOI: 10.1016/j.atherosclerosis.2018.04.037.

Weng SF, Kai J, Andrew Neil H, et al. (2015) Improving identification of familial hypercholesterolaemia in primary care: Derivation and validation of the familial hypercholesterolaemia case ascertainment tool (FAMCAT). Atherosclerosis 238(2):336343 DOI: 10.1016/j.atherosclerosis.2014.12.034. 
Wilson BJ, Qureshi N, Santaguida P, et al. (2009) Systematic review: Family history in risk assessment for common diseases. In: Annals of Internal Medicine, 151(12):878-885 DOI: 10.7326/0003-4819-151-12-200912150-00177. 


\section{Box 1 - Pharmacogenomic issues.}

\section{Discovery research}

This includes assessing genotype-phenotype associations, characterising pathogenic variants and identifying patients at higher risk of disease due to their genomic variation.

\section{Clinical validation}

This includes research to identify the effects of genomic information on health outcomes and the clinical utility of genomic testing in various clinical scenarios. Clinical utility is an important concept in this context and is defined as "the relevance and usefulness of an intervention in patient care" (Lesko et al., 2010). In primary care this is multi-dimensional, involving physical, psychological and social dimensions.

\section{Clinical implementation}

How best to embed routine genomic testing in clinical care, what information technology systems are needed to support this and how best to educate healthcare professionals and patients?

Source: Manolio et al., 2019 